\title{
3D VISIBILITY ANALYSIS IN URBAN ENVIRONMENT - COGNITION RESEARCH BASED ON VGE
}

\author{
T. P. $\operatorname{Lin}^{\mathrm{a}} *$, H. Lin', M. Y. $\mathrm{Hu}^{\mathrm{b}}$ \\ ${ }^{a}$ Earth Sys and Geo Info Science, The Chinese University of Hong Kong, Sha Tin, N. T., Hong Kong - \\ lin_tp@cuhk.edu.hk \\ ${ }^{\mathrm{b}}$ Shenzhen Research Institute, The Chinese University of Hong Kong, Shenzhen, Guangdong, China - (huilin, \\ humingyuan)@cuhk.edu.hk
}

ISPRS 3DGeoInfo Conference 2013

KEY WORDS: 3D Visibility Analysis, Virtual Geographic Environment, Urban Environment, Human Perception, Visual Field

\begin{abstract}
:
The author in this research attempts to illustrate a measurable relationship between the physical environment and human's visual perception, including the distance, visual angle impact and visual field (a 3D isovist conception) against human's cognition way, by using a 3D visibility analysis method based on the platform of Virtual Geographic Environment (VGE). The whole project carries out in the CUHK campus (the Chinese University of Hong Kong), by adopting a virtual 3D model of the whole campus and survey in real world. A possible model for the simulation of human cognition in urban spaces is expected to be the output of this research, such as what the human perceive from the environment, how their feelings and behaviours are and how they affect the surrounding world.
\end{abstract}

Kevin Lynch raised 5 elements of urban design in 1960s, which are "vitality, sense, fit, access and control". As the development of urban design, several problems around the human's cognitive and behaviour have come out. Due to the restriction of sensing knowledge in urban spaces, the research among the "sense" and the "fit" of urban design were not quite concerned in recent decades. The geo-spatial cognition field comes into being in 1997 and developed in recent 15 years, which made great effort in way-finding and urban behaviour simulation based on the platform of GIS (geographic information system) or VGE. The platform of VGE is recognized as a proper tool for the analysis of human's perception in urban places, because of its efficient 3D spatial data management and excellent $3 \mathrm{D}$ visualization for output result.

This article will generally describe the visibility analysis method based on the 3D VGE platform. According to the uncertainty and variety of human perception existed in this research, the author attempts to arrange a survey of observer investigation and validation for the analysis results. Four figures related with space and human's perception will be mainly concerned in this proposal: openness, permeability, environmental pressure and visibility, and these will also be used as the identification for different type of spaces. Generally, the author is aiming at contributing a possible way to understand the reason of human's cognition in geo-spatial area, and provides efficient mathematical model between spatial information and visual perception to the related research field.

\section{INTRODUCTION AND RESEARCH BACKGROUND}

\subsection{General Instructions}

$3 \mathrm{D}$ visibility analysis is mainly discussed in this article, which is also related with the relationship between the space types and human's visual perception in a 3-dimensional research scale. The initial idea of this research originates from the research progress of Kevin Lynch in 1981, where he has listed five basic elements for urban planning (Lynch 1981), including vitality, sense, fit, access and control. These elements supply a guide for urban design and relatively efficient value standards. In these five elements, "Sense" of urban space is described as the perception or mental structure received by urban users, and another word "fit" means the form and capability of urban space fit the behaviour of human being. However, during current research the field related with human perception has not been concerned quite much, since it is thought to be difficult to deal with human's mental world. One reason is that, the research around human perception is considered hard to be measured or quantified, which restricts the analysis between the environment and human's perception.

The visual perception of human is often thought to be the most significant issue among all types of human's perception. All the human senses of urban spaces come from urban image of the observers, which are considered to be various from each person. Human's knowledge background and activity with urban space will affect and generate different sense from standard meaning (Montgomery 1998). In the past there were not quite much psychological investigation related with metal images of geographic space, and all the efforts for psychologists mainly focused on the physics and physiology of the senses in laboratory work (Gould 1973). After the conception of isovist and viewshed were raised by several scholars (Tandy 1967, Benedikt 1979, Lynch 1976), the topic between space and human's visual sense starts to be introduced as the visibility analysis into the research field, and most of the researchers tend to focus on the common sense of the observer generated by the located environment. As a result of development of 3D

\footnotetext{
* Corresponding author.
} 
technology and visibility analysis, the idea of 3D visibility analysis has come into being, which mainly deals with the visibility computation for urban area in 3D scales.

After the introduction of Geographic Information Science (GIS) and Virtual Geographic Environment (VGE) technologies, modern analysis methods have been playing an important role in the field related to urban environment in recent few decades. Not only for the basic analysis aspects such as the information management, road network analysis and routing, GIS begins taking part in the research of visibility analysis via the tool of DEMs (Digital Elevation Model) (Yang et al 2007, Morello and Ratti 2009, Suleiman et al 2011). VGE provides an efficient 3D based platform for the data access, storing and management, and a friendly interface accepted by final users, which makes the geographic output result lively and comprehensive. Due to its full 3D visualization scenes, VGE is thought to be an available platform for the visibility analysis, and also capable in the complex urban environment with quantities of buildings. Due to these reasons, the 3D VGE platform is adopted as the place for the extraction of building spatial relationship, the 3D visibility analysis and correlation study with human's perception in this article.

\subsection{Current Visibility Analysis Progress and Limitations}

The conception of visibility (or visual, visual perception) analysis was raised very early. In some traditional practices the views of pedestrians walking in the urban open space were recorded through photographs or sketches in order to understand the visual effects (Yang et al 2007). In the 1970s, two main approaches emerge in the visibility analysis context: the concept of viewshed in terrain and landscape analysis and the concept of isovist in architecture and urban space (Leduc et al 2011). Benedikt (1979) was the first who systematically introduced the isovist, the area visible directly from any location within the space. According to current research progress, numbers of researchers showed their interests in the research of isovist and found a few new ways for the acquisition of spatial indexes from the isovist idea, which could be applied to describe the figures like space volume, openness, and visibility (Turner and Penn 1999, Turner et al 2001, Stamps III 2005a, Stamps III 2005b). The other one viewshed analysis, in the opinion of Llobera (2003), is efficient in recent GIS applications since it could be acquired from connecting the grid cells in a digital elevation model (DEM) with the location of viewpoint as the line of sight within any specified distance. The idea has been widely adopted in recent viewshed or isovist studies, which is common in the research field of $2.5 \mathrm{D}$ and $3 \mathrm{D}$ visibility analysis. Currently the GIS-based viewshed analysis provides a full $360^{\circ}$ horizontal view orientation, and $0^{\circ}$ to $90^{\circ}$ vertical view orientation.

Based on the knowledge of 2D isovist and viewshed, several approaches for 3D visibility analysis has been carried out in recent 8 years, and numbers of automated models have been developed as the efficient analysis tools. Several researchers have developed the method as viewsphere (Yang et al 2007) or view-oriented 3D visibility analysis model (Fisher-Gewirtzman and Wagner 2003, Fisher-Gewirtzman et al 2005) for dealing with the visual effects in urban environment. Besides, the idea of DEM or TIN models are widely used because of its suitable feature for visibility analysis (Morello and Ratti 2009, Suleiman et al 2011).
Referring to the research attempts done by other researchers, although visibility analysis in $2 \mathrm{D}, 2.5 \mathrm{D}$ and $3 \mathrm{D}$ has achieved fruitful goals, some problems are still remaining to be solved in the near future. These may include: (1) Lack of full 3D scene for visibility analysis, since even DEM or TIN based visibility analysis can only be recognized as $2.5 \mathrm{D}$; (2) Lack of consideration about areas below horizon, since most of the researchers supposed the experiment site to be located on a flat plane and neglect the negative visual angles; (3) Lack of combination of terrain and buildings / urban environment, and most researches are integrating the two features together for the data procession; (4) Lack of consideration about human agent, as rare of the researchers designed the experiment with a movable view point, or represent it by a human agent. According to this situation the author intends to design a method for $3 \mathrm{D}$ visibility analysis to overcome the barrier in current research, and provide a reference to the related research fields.

\section{METHODOLOGY AND EXPERIMENT PREPARATION}

\subsection{Inner Structure of the VGE Platform}

Comparing with 3D GIS which is also an expert platform dealing with terrains, urban environment and building models in 3D scales, the virtual Geographic Environment (VGE) has some special features while dealing with geographic problems. In this experiment, VGE acts more alike a platform integrating all types of data source with friendly interface, and can support or be accessed by other software. The functions such as data storing, management, analysis and visualization could be realized in this platform by inputting data into VGE. The software of Open Simulator (OpenSim for short, website: http://opensimulator.org/) together with Second Life Viewer will be adopted as the platform of VGE.

\subsubsection{Input, Storing and Management of 3D Spatial Data:} Due to the MySQL based 3D spatial information management feature, application for the Second Life engine (website: $\mathrm{http} / / /$ secondlife.com/) and a few 3D related research progresses (Friedman et al 2007, Kinicki and Claypool 2008), the OpenSim has been chosen and adopted as the platform software for this experiment. The 3D geographic information are able to be handled by OpenSim, including the input and storing of terrain (via DEM), building models and vegetation, and visualized through the its viewer for the final presentation. According to the operation structure of OpenSim, two parts as server and customer are separated for different functions, which are normally operated by two or more computers (Figure 1).

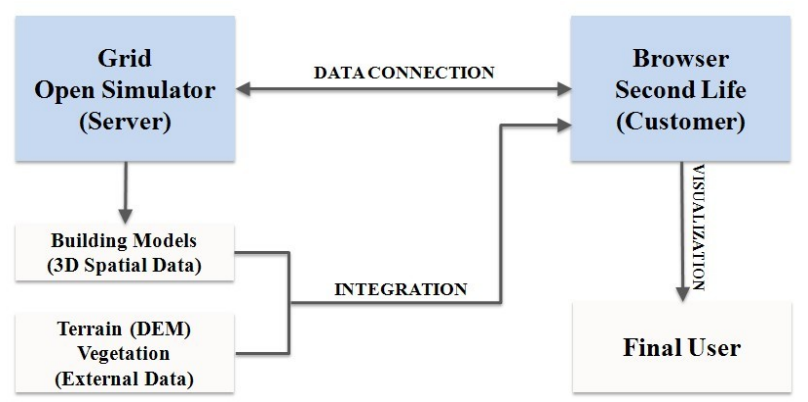

Figure 1. Inner Structure of OpenSim - The VGE Platform Used in this Experiment 
The function of Open Simulator Grid is for the data storing, procession and management, which contains the spatial information for terrain and building models. The other one, the browser of Second Life, is in charge of the data input (via building model construction or importing from external) and visualization, which directly faces to the final user. For 3D visibility analysis, the required information as building models is acquired from the database, which belongs to the part of server. Necessary external data such as DEM for terrain will be integrated with the fetched model for more detailed analysis. Moreover, it is possible to use the integrated information by the browser for the final visualization.

\subsubsection{Computation of 3D Spatial Data:}

OpenSim provides a platform for 3D spatial data input, storing and management, but it is not suitable for the computation with these data sources due to its inner structure. As the existence of MySQL based database, the spatial data stored inside by OpenSim could be extracted and processed through other programs, only if it has the ability of reading the information from the database. Currently the author applies Python as the program language tool for the extraction and computation, and a website based on Python / Django is acting as the data engine and interface presenting to the user who are the controllers of the computation. Not only the data source stored inside the OpenSim database, but also external data in MySQL format, such as the pollutants or noise distribution data handled by other software, could be integrated in this platform and finally applied for the visualization according to the experience of the author's group. Besides, as the DEM data is treated as a type of external data in this research, the import and computation are also handled by the program via MySQL database for the further data integration.

\subsubsection{Visualization and Expression of 3D Spatial Data:}

After the procession of $3 \mathrm{D}$ spatial data for visibility analysis, the visualization and expression of the output needs to be handled via the VGE platform - this is the work of the customer viewer as Second Life (Figure 2). Normally the data sources for the visualization are from OpenSim Grid (server), but necessary plugins for external data sources are in demand in this step, because of unsupported external sources by the customer viewer (browser). Proper modification is done and plugins are added to the Second Life viewer, including the part in charge of rendering specified data source reading from MySQL database. According to the special features of data source, colourful points, lines, planes and transparent boxes are adopted for the final result representation due to these input sources.

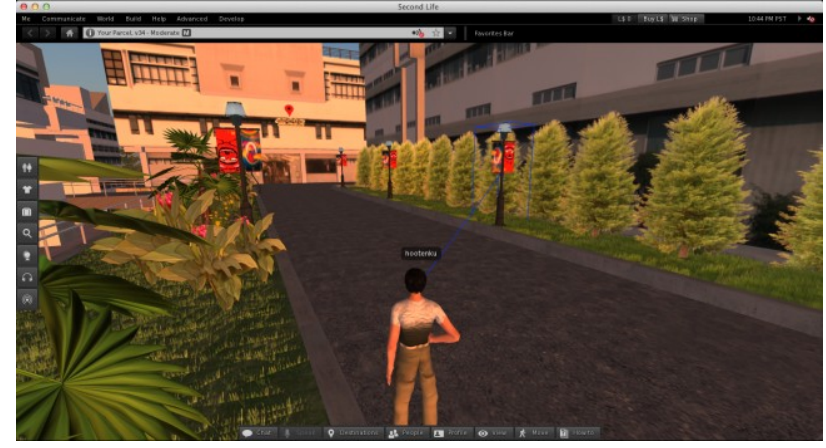

Figure 2. A Brief View of the 3D Based VGE Platform Used in this Experiment - OpenSim and Second Life Viewer;

This Picture Shows the Extraction Procession for the Visual Angle of Models inside the Platform

\subsection{D Visibility Analysis Model}

As an extension function of OpenSim based VGE, the author is trying to develop a tool for visibility analysis in urban environment, which is called " $3 D$ Visibility Analysis Model" in this article. The general idea of this model is: from the spatial distribution of different types of terrain, buildings, city furniture and vegetation, the computation of spatial relationship could be processed and applied for correlation study with human's feeling. Two more issues are discussed in the following paragraphs as:

\subsubsection{Data Sources:}

According to the various features among the target objects, 3 types of data sources are considered in this experiment, which are terrain, buildings and vegetation (Figure 3).

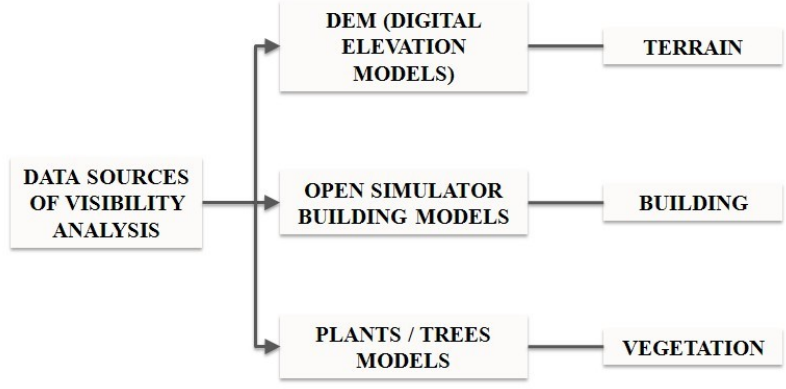

Figure 3. Integration of 3 Types of Data Sources in the 3D Visibility Analysis Model

For the representation of terrain, a $0.5 \mathrm{~m} * 0.5 \mathrm{~m}$ resolution DEM (Digital Elevation Model) is adopted for the computation of viewshed for specified viewpoint. The pixel-based data arrangement adapts properly for the viewshed analysis and result storing based on MySQL database. The second step, the author extracts the building models from the OpenSim Grid, for the representation of building data of the reality. Based on the platform of OpenSim, the whole campus of CUHK has been virtualized into the server with enough precision for the building locations, scales and textures, which meets the data source requirements of this experiment. The extraction of buildings' spatial data is processed by Second Life Viewer, which is a customer viewer for the $3 \mathrm{D}$ building models visualization and do the exportation during the rendering process. The final issue, the vegetation is thought to be an additional data source for the visibility analysis, according to the widely distribution of vegetation inside the campus. Currently there is not any good method for the representation of vegetation due to its complexity, and it still remains to be discussed in the author's further progress. These 3 types of data sources process separately in the visual data computation, and the integration is designed afterward for the analysis of visibility features.

\subsubsection{Features Concerned:}

For the 3D visibility analysis model in this article, 3 features are mainly concerned here, which are visual distance, visual angle and visual field. These features are the first group of outcomes in the visibility analysis, relatively significant for the data source of building models in OpenSim. The visual distance is defined as the distance in 3D from the observer towards a target object, and it could be calculated if the coordinates of the viewpoint and target are known. The visual angle (for single building) in this article is divided into two categories as 
horizontal and vertical, for the representation of object visible part in those two mutually perpendicular directions. As the location and shape of the building is known, the visual angle calculation for a specified viewpoint turns to be possible. The last factor visual field, whose definition is the distribution of visual angle for surrounding objects in all directions $(360$ degrees), could be drawn from the visual angle data collected for visible objects. It could also be treated as the viewpoint skyline spectrum for the surrounding objects. These mathematical figures could be applied in founding the relations with human's visual perception, such as the feeling of crowd, order, continuous or even emotional feelings as comfortable and harmonious, as this result, they are our initial concerned factors in this visibility analysis approach.

\subsection{Experimental Sites in Reality - CUHK Campus}

The experiments take place in the campus of CUHK (the Chinese University of Hong Kong), which occupies an area of $1.34 \mathrm{~km}^{2}$ and includes over 80 single buildings. Because CUHK is located on a hillside with the altitude range from $5 \mathrm{~m}$ to $170 \mathrm{~m}$, compared with other same area places, it contains more abundant topography types obviously. The various terrain and buildings in CUHK supply relatively suitable experimental sites to this research, as most of the representative space types can be discovered in the small campus. Due to the level of enclosure, openness and visibility, there are four main types of building groups in CUHK, which are defined as squares, courtyards, terraces and streets (Details in 2.4). The distribution of specified group of sites could be referred to the Figure 4 . Based on the spatial information extracted from the VGE models as visual distance, visual angle and visual field, the four figures like openness, permeability, visibility and environment pressure will be processed for the identification to distinguish each space type.

Although in CUHK there are abundant space types, it also indicates a few existed problems that are distinct from other outside places. According to the terrain and building groups types in CUHK, several special circumstances have been listed as following:

2.3.1 A new space type of half-enclosure as "terraces" commonly exists inside the campus.

The terraces, which are often recognized as the flat spaces on the hillside, refer to the places surrounded by buildings or walls in no more than two directions and separated from the other space through its special topography. Few researches have been down due to the rarity of this space type, but here it would be considered to have the same importance as squares or courtyards, because of its commonness in the campus of CUHK. Besides, as most of CUHK buildings are facing to the coast, there are a numbers of roof terraces in the campus, which also belong to this space type.

2.3.2 Some target objects are long distance away from observer, whose effect towards visual sense is slighter than closer ones.

This situation is common inside the campus because of its mountainous topography, such as the a few hill tops locating far away from the campus. These places could be seen during good weather but often disappear due to the unusual situation of low visibility (fog, haze or heavy rain). Besides, the insufficient contrast of the scene could also decrease the influence of these objects. However, to reduce the uncertainly of weather issues, only good weather is considered in this experiment but effects of distance are considered to be added as a special issue.

\subsubsection{Negative vertical visual angle may be existed} because of the hilly topography.

As most of the past studies have only concerned the visual angle above the horizon, the scene below our visual line hasn't been considered. For the hilly topography inside the campus of CUHK, this situation becomes obvious and urgent in the study of this proposal. For instance, several terraces such as the platforms of several teaching buildings, the observer standing on the platforms can easily get the visual image from low altitude area, even a few of lower buildings would get into the scene and influence the person.

2.3.4 Vegetation issue is also significant in the campus during the visibility analysis.

Because of the high coverage of vegetation inside campus, the effect of vegetation cannot be neglect during this research. They play a very important role in the space enclosure inside campus and are significant for the enclosure of streets. Besides, the trees and bushes often exist and separate two close spaces, which make the empty space full of interests.

\subsection{Classification of Space Types for Experimental Sites}

According to the research progress of Ashihara 1975, the author used phrases as "squares", "courtyards" and "streets" to describe the space openness in his research. Similar with that research, the classification of space types in this article is justified to the level of enclosure but with "terraces" added. According to the definition of 4 space types (Squares, Courtyards, Terrace and Streets) introduced, the author will select 40 points from CUHK campus and divided them into these 4 groups. The classification of space types shows a relationship as following table (Table 1):

\begin{tabular}{|c|c|c|c|}
\hline \multirow{4}{*}{$\begin{array}{l}\text { Space } \\
\text { Types }\end{array}$} & \multirow{2}{*}{ - Enclosure } & $\begin{array}{l}\text { - Square } \\
\text { (Open type) }\end{array}$ & $\begin{array}{l}\text { E.g. Central Campus } \\
\text { Square } \\
\text { Square in front of } \\
\text { MTR Station }\end{array}$ \\
\hline & & $\begin{array}{l}\text { - Courtyard } \\
\text { (Closed Type) }\end{array}$ & $\begin{array}{l}\text { E.g. Backyard of } \\
\text { Science Centre } \\
\text { Courtyard of Lady } \\
\text { Shaw Bldg. }\end{array}$ \\
\hline & \multirow{2}{*}{$\begin{array}{l}\text { - Half- } \\
\text { enclosure }\end{array}$} & $\begin{array}{l}\text { - Terrace } \\
\text { (Two Adjacent } \\
\text { Boundaries) }\end{array}$ & $\begin{array}{l}\text { E.g. Platform of } \\
\text { Esther Lee Bldg. } \\
\text { Platform of S. H. Ho } \\
\text { College }\end{array}$ \\
\hline & & $\begin{array}{l}\text { - Street } \\
\text { (Two Opposite } \\
\text { Boundaries) }\end{array}$ & $\begin{array}{l}\text { E.g. Central Avenue } \\
\text { Road next to Tin Ka } \\
\text { Ping Bldg. }\end{array}$ \\
\hline
\end{tabular}

Table 1. Classifications of Space Types in this Proposal

At the very beginning all spaces are divided into two main types, the one of enclosure spaces and the other of half-enclosure spaces. For the first type, the word "enclosure spaces" refers to the spaces totally surrounded by boundaries, which may include walls, buildings, terrains (hillside) or vegetation. A few gaps may exist in this type of space, but they are not wide enough to interrupt the enclosure property of the whole space. Squares and courtyards are considered to be two different space types under the definition of enclosure spaces. For the other one, the half- 
enclosure space contains the space types with boundary surrounded not in the entire perimeter. This type includes streets and terraces that are normally have only two or three boundaries, either in opposite or adjacent directions.

\section{CURRENT PROGRESS, EXPERIMENTS AND RESULTS}

Based on the platform of Open Simulator (a VGE platform), a set of experiments have been designed for the 3D visibility analysis model. These include the attempts in the following 5 sections, from the selection of information collation in experimental sites to the data processing methods.

\subsection{Information Collation of Experimental Sites}

Information collation of experimental sites is necessary at the beginning of the experiment. As mentioned in Section 2.3 of this article, the author has picked up 40 points from CUHK campus to represent the 4 types of space, with each 10 points for squares, courtyards, terraces and streets. Several efforts have been made before the experiment is taken, including the investigation of site basic information and collation. Pictures, geographical location and a brief description have been prepared for each experimental site. The detailed information as the locations of those 40 points could be referred to Figure 4 .

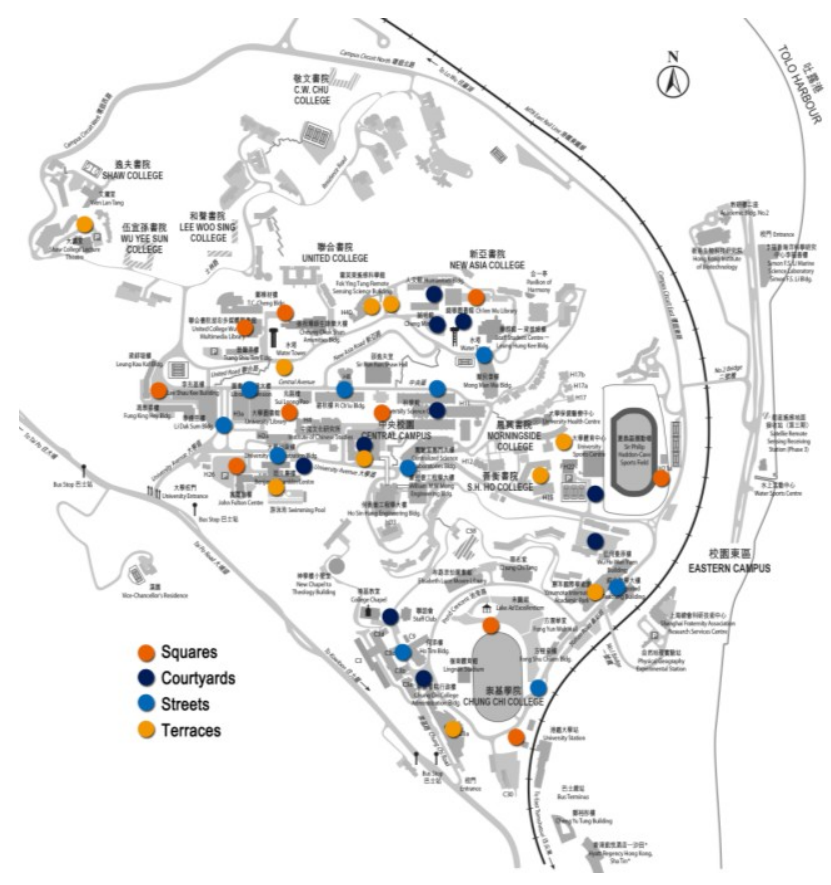

Figure 4. Experimental Sites Distribution in CUHK Campus, and Categories of 40 Selected Points

Although the topography in CUHK is diverse enough to find sufficient places to meet the classification requirement in this article, there are still a few problems remaining to be solved in the author's further progress. Take the streets for instance, the hilly terrain of CUHK limited the length of straight street, which makes the experiment lack of the spaces with regular, orderly and continuous street façade. Besides, terraces as a special feature for hilly campus have been added into the space types, and they will be on behalf of the visibility analysis in a special space type that has never been explored before. Because of this, the examples of the points in CUHK may not be representative enough for each space type, and extra experiment may be designed in future to validate the accuracy of the classification method.

\subsection{Spatial Relationship Figures: Visual Distance, Visual Angle and Visual Field}

For initial data process of 3D visibility analysis 3 figures are considered to be significant, that is the visual distance, visual angle and visual field mentioned in Section 2.2.2. These three are important because they define the geometric issues of space. Distance, that means the distance from the observer (viewpoint) to the boundary, is controlling the width scale of the opening space. Once the distance is getting smaller, most of the observer would feel the surrounding space more crowed, narrow and even pressured, which is thought to be the common sense of human being. The figures of visual angle are decided by the height of surrounding buildings, which indicates the visual significance or impact caused by the target objects. For the same building, different visual angle may be generated if the distance is changing, and for larger distance between viewpoint and target, the visual angle will become smaller. Larger visual angle indicates that the object could be more easily perceived by the observer, which means more significant or impact towards the human being's sense. If considering the surrounding visual angle distribution as a total, the idea of visual field comes into being. As the visual field is decided by the surrounding environment, it defines the space type of the urban environment. Moreover, several sorts of human perception are closely related with the type of visual field, such as openness, permeability, visibility and environment pressure.

\subsubsection{Visual Distance:}

It is proved that human's two eyes could generate a full 3D scene of the world because there's slightly visual direction difference between the images percept by the two eyes, and that's why the distance has been noticed as one of the significant figures here. Different distance will affect the variance in visual perception, such as the change of openness and intimacy. Visual distance could be conveniently drawn out from either DEM or 3D building models. As the coordinates of both viewpoint and target are known, the distance is get from the distance formula in three-dimensional space as Formula 1:

$$
d=\sqrt{\left(x-x_{0}\right)^{2}+\left(y-y_{0}\right)^{2}+\left(z-z_{0}\right)^{2}}
$$

where $\quad d=$ distance between viewpoint and target
$x_{0}, y_{0}, z_{0}=$ coordinates of viewpoint
$x, y, z=$ coordinates of target

Visual distance is thought to be the one significantly influencing human's openness perception. During the research result of Bittermann and Ciftcioglu 2006, the relationship between distance of noticing flat object and openness perception is described by a sigmoid function, that is, the openness feeling normally increases with distance gradually at the beginning, sharply around certain value and tends to be steady finally. Another figure may relate with distance is visual impact. It is not similar with openness, which concerns more about how important an object could be perceived by human being and acts inversely proportional to the distance. Those would both be considered in the visibility analysis process. 


\subsubsection{Visual Angle:}

Visual angle is another significant issue because it decides the visual impact of the target. It is considered that larger visual angle attracts human's attention more easily, which means larger visual impact for the target object. Visual angle in this research is divided into two smaller groups for better representation in 3D world, and they are visual angle in horizontal and vertical direction. The visible area of the target object could be formed by integrating the two types of visual angle value together. The horizontal visual angle is valued from $0^{\circ}$ (to the north) and increasing clockwise to $359^{\circ}$, and the vertical visual angle is ranged from $-90^{\circ}$ to $90^{\circ}$, with $0^{\circ}$ referring to the horizon.

Similar with the process for distance computation, the calculation of visual angle must be done both for DEM (terrain) and building models. For DEM, the visual angle for each pixel is processed from the coordinates of viewpoint and target pixel like the following formula, and all the visual angle value will be changed from radius to degree:

$$
\begin{aligned}
& v_{h}=\arccos \frac{y-y_{0}}{\left(x-x_{0}\right)^{2}+\left(y-y_{0}\right)^{2}},\left(y>y_{0}, y=y_{0}\right) \\
& v_{h}=\arccos \frac{y-y_{0}}{\left(x-x_{0}\right)^{2}+\left(y-y_{0}\right)^{2}}+\pi,\left(y<y_{0}\right) \\
& v_{v}=\arctan \frac{z-z_{0}}{\left(x-x_{0}\right)^{2}+\left(y-y_{0}\right)^{2}+\left(z-z_{0}\right)^{2}}
\end{aligned}
$$

$$
\begin{array}{ll}
\text { where } & v_{h}=\text { horizontal visual angle } \\
& v_{v}=\text { vertical visual angle } \\
& x_{0}, y_{0}, z_{0}=\text { coordinates of viewpoint } \\
& x, y, z=\text { coordinates of target }
\end{array}
$$

For building models, a method of bounding box is applied in this experiment, that is to use a 3D box tightly contained the irregular building model and do the visual angle calculation for box only (Figure 5). For complex buildings groups of boxes will be used, making sure that each small box is only in charge of containing for a part of the building, and do the result integration afterward. By calculating the visual angle value for each vertex, the range occupied for each building will be clear. Currently only the maximum and minimum visual angle in both horizontal and vertical is considered. The improvement is necessary for better representation of building visual angle distribution.

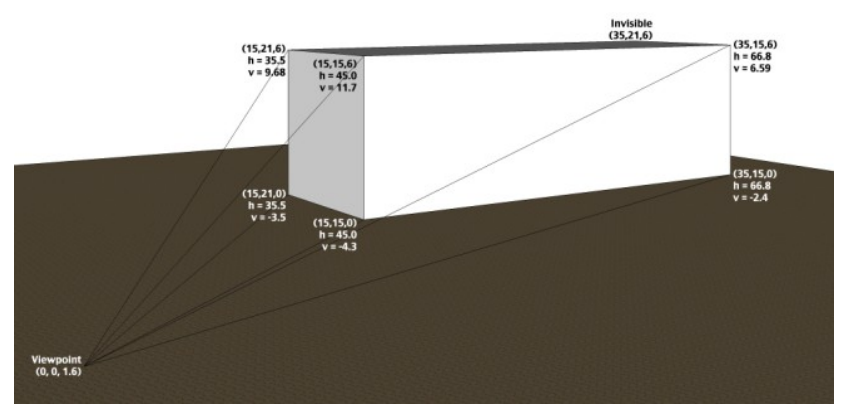

Figure 5. Visual Angle Computation Method for Buildings: Bounding Box is used and calculation is for each vertex, only max and min of horizontal or vertical value is considered

\subsubsection{Visual Field:}

Visual field can be explained as the combination of a set of spatial visual data for a specified viewpoint, which is based on the idea of 3D isovist with expression method changed. It could be recognized as the integration of distributed visual distance and visual angle in every direction, and is closely connected with the space type and potential influence with human's perception. As just mentioned, the visual distance and visual angle will be contained and dealt separately in the generation of visual field. The distribution of distance for the viewpoint indicates the openness of the whole environment, and the other of visual angle indicates the skyline and horizon of surrounding. This spectrum-liked visual field could provide a quantified data source for the analysis of images in the human's mental mind, which is perceived by the eyes.

Different visual field pattern will imply different spatial types. Take the latter one, the vertical visual field composed by visual angle for instance, for the square-liked pattern, the visual angle surrounding by the observer should be more or less the same and limited no more than a certain value; for courtyards the visual angle value is larger, but also controllable. Because the squares and courtyards are belonging to enclosure space types, normally there won't be any wide "gaps" between each visual angle bar. But this would be different from the half-enclosure spaces, such as streets or terraces. For streets the bar of visual angle are high enough with two obvious gaps in two opposite directions; for terraces the visual angle bar is similar but with wider, more continuous gaps in between. These also could be inferred from the percentage distribution of visual angle value. Same information could be extracted from the distance visual field, which is often introduced by $2 \mathrm{D}$ isovist theories. Moreover, even for the same space type, the openness, enclosure or permeability is affected by the level or distribution of visual angle value. For enclosed space the visual angle is relatively higher than open space; and for streets the width of the "gaps" decides the permeability of the whole environment. As a result, the visual field spectrum indicates different pattern with each other but similar with the same type of urban spaces, which could be extracted through the disciplines of spectrum.

\subsection{Visual Data Computation for Terrain: by Using DEM}

According to the data source layers introduced in Section 2.2.1, visual data computation is processed separately for terrain and buildings. DEM of CUHK Campus area is used for the representation of terrain, with a fine resolution of $0.5 \mathrm{~m} * 0.5 \mathrm{~m}$. This magnitude of resolution should meet the requirement of the experiment. DEM and TIN based visibility analysis is often thought to be the initial study for the environmental spatial relationship in the 3D scales. According to the methods developed by the former scholars (Fisher-Gewirtzman et al 2005, Yang et al 2007, Morello and Ratti 2009), viewshed or isovist extraction has often been done based on the data sources of DEM. These progresses give the author some suggestion about the computation of visual data in the current progressing experiment.

In this research, the pre-processed DEM data is saved as a matrix of height values, with each height value coordinated with the axis value (Figure 6a). The coordinates of the experimental sites have been collected before the calculation starts, and with these figures the 3D isovist could be extracted by the process introduced in Morello and Ratti's research. That is, to calculate the visual distance, visual angle and direction between each of the DEM pixels and the viewpoint, and to do the comparison 
work between the visual angles if two directions are the same. The largest visual angle value will be retained and marked on the map as the 3D isovist for the target site, and the distance would be saved together with the visual border point (Figure $6 \mathrm{~b}$ $6 c)$. This data procession would be similar with that with building models, and visual distance, visual angle and visual field will be computed and stored sequentially in order to intergrade with the result for buildings. After the extraction for a series of points in 360 degrees direction, the visual field of the viewpoint locating in each experimental site will be clear.
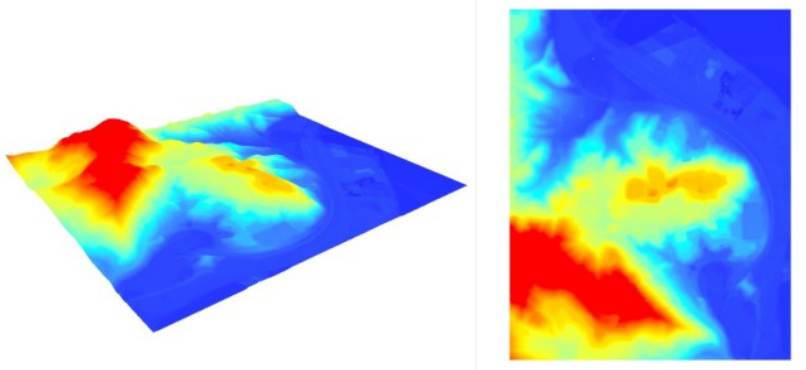

(a)

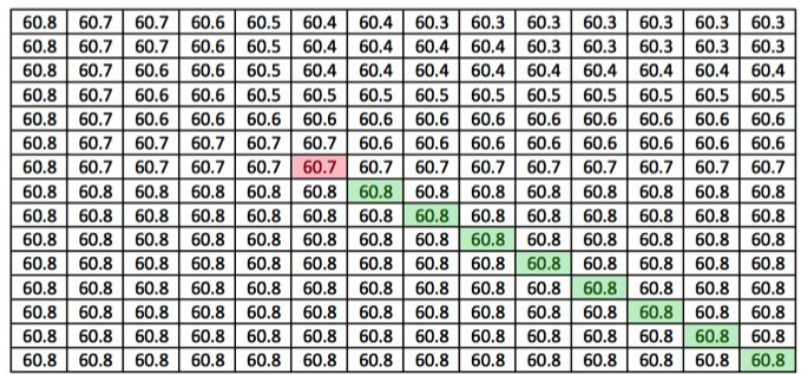

(b)

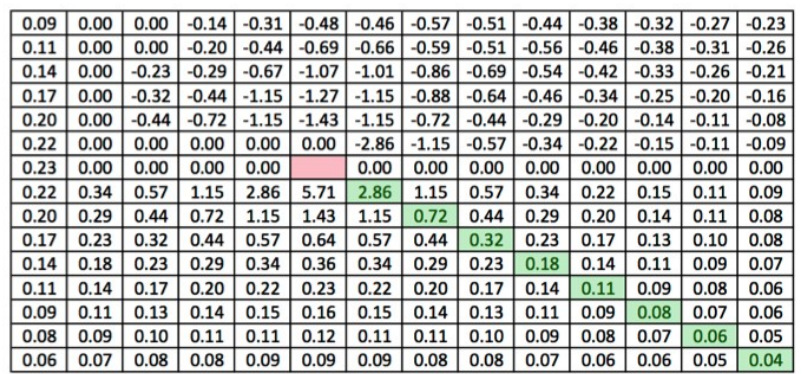

(c)

Figure 6. (a) DEM Used in this Experiment (CUHK Campus, $0.5 \mathrm{~m} * 0.5 \mathrm{~m})$; (b) DEM (Height Matrix): Use the algorithm to calculate the visual data for each pixel, and do the comparison; (c) Visual Angle for DEM: For direction 135, take max value of 2.86 as the visual angle for Point Red (E.g.)

The visual data result for terrain cannot be used for visibility analysis directly. For a specified view, terrain, buildings and vegetation compose the scene of the surrounding environment, and they cannot be separated from each other. As the DEM data only represents the geographical visible area of terrain for a certain viewpoint, and there may be other shelters (such as the buildings) narrowing the visible area, especially for the viewpoint locating in a crowded environment.

\subsection{Visual Data Computation for Buildings: by Using Models from OpenSim}

The steps for building representation in 3D VGE platform are more complicated than terrain, because of the complexity of building shapes, structures and textures. In this article, OpenSim models have been applied for the shape representation of buildings, and used for the visual data computation. As there are around 80-100 buildings in CUHK campus, computation for each building can only be done one by one. According to a specified scene (a viewpoint in one experimental site) Visual distance, visual angle and visual field calculation are done for every building and building group.

To avoid inaccurate computation, in this experiment building models are required to be divided into smaller parts, as most of the buildings are in irregular shapes and cannot be dealt as a total. Smaller parts for the building will reduce the unnecessary error in the spatial information extraction. During the procession a bounding box restricts each part of the building, with each vertex on the building part strictly limited inside the surface of the box. The distance between the observer and the target could be got from the closest vertex and the location of viewpoint. The other figure is the building view angle, which is decided by the maximum and minimum value of vertex generated visual angle value in both horizontal and vertical direction. Although this method is still not accurate enough, it is thought to be capable for current visibility analysis.

This process should be done for every visible building inside a certain scene, and normally there won't be over 20 visible objects in the same scene. Taking the site of Central Campus Square (P1) for example (Location as Figure 7), the author has arranged the viewpoint located in between Sir Run Run Shaw Hall and Lady Shaw Building. There are total 18 objects (buildings) visible from this point, and they are shown according to the following Table 2 . In this table I have listed the distance and visual angle figure for all visible buildings extracted from the VGE platform. For instruction, the visual angle for a single building is expressed by a group of 4 figures, which are the maximum and minimum of angle in horizontal direction, and the maximum and minimum in vertical direction.

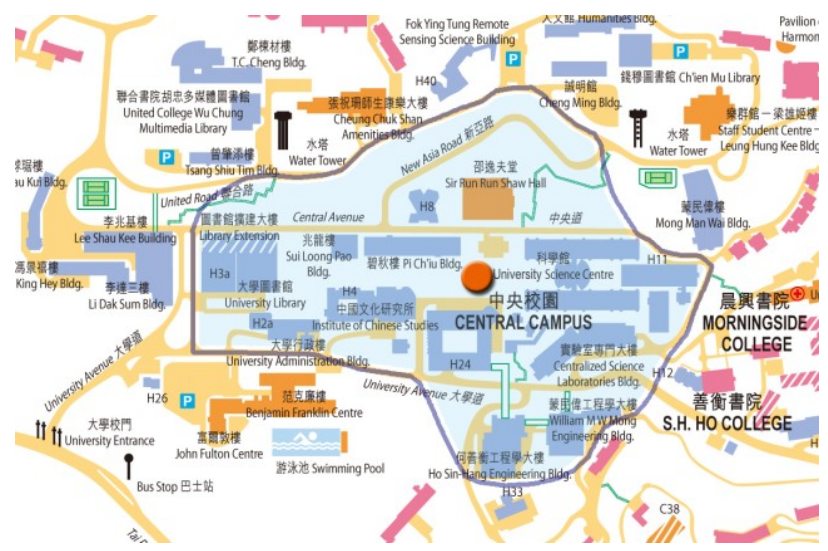

Figure 7. Map of Visible Area for P1 - Central Campus Square, Which Also Shows the Location of Each Surrounding Building;

The View Point is in the Center and Marked with Orange

\begin{tabular}{|c|c|c|c|c|c|}
\hline \multicolumn{6}{|c|}{ P1 - Building Visibility Figures } \\
\hline Name of Buildings & $\begin{array}{c}\text { Avg. } \\
\text { Dist }\end{array}$ & \multicolumn{2}{l|}{ Visual A.H. } & \multicolumn{2}{l|}{ Visual A.V. } \\
\hline $\begin{array}{c}\text { FYT Remote Sensing } \\
\text { Science Bldg. }\end{array}$ & 175.59 & 3.47 & -21.62 & 23.36 & 9.17 \\
\hline Sir Run Run Shaw Hall & 52.53 & 27.39 & -14.89 & 16.87 & -1.21 \\
\hline
\end{tabular}




\begin{tabular}{|c|c|c|c|c|c|}
\hline Cheng Ming Bldg. & 212.43 & 31.36 & 16.70 & 16.41 & 9.83 \\
\hline Ch'ien N & 9.94 & 38.15 & 28.48 & 13.68 & 9.12 \\
\hline NA Wa & 2.47 & 49.79 & 46.50 & 2.21 & 11.09 \\
\hline Charles K. & 5.76 & 85.45 & 48.05 & 26.53 & -0.05 \\
\hline $\begin{array}{l}\text { Science Centre } \\
\text { Lecture Hall }\end{array}$ & 3.08 & 100.92 & 79.86 & 15.72 & -0.44 \\
\hline Ma Lin Bldg. & 46.48 & 132.71 & 94.93 & 26.36 & -7.96 \\
\hline $\begin{array}{l}\text { Centrali } \\
\text { Laborat } \\
\end{array}$ & 57.76 & 153.80 & 100.97 & 12.45 & -11.29 \\
\hline $\begin{array}{l}\text { William M W Mong } \\
\text { Engineering Bldg. }\end{array}$ & 151.45 & 156.67 & 141.43 & 0.87 & -18.02 \\
\hline $\begin{array}{c}\text { Ho Sin-Hang } \\
\text { Engineering Bldg. }\end{array}$ & 136.05 & 177.76 & 151.32 & 6.72 & -14.95 \\
\hline Lady Shaw Bldg. & 19.71 & 234.77 & 147.91 & 5.36 & -20.05 \\
\hline $\begin{array}{c}\text { Institute of } \\
\text { Chinese Studies }\end{array}$ & 64.46 & 263.70 & 217.87 & 14.00 & -14.28 \\
\hline $\begin{array}{c}\text { Beacon and } \\
\text { Gate of Wisdom }\end{array}$ & 66.07 & 273.11 & 266.89 & 1.29 & -0.83 \\
\hline University Library & 194.47 & 275.60 & 271.27 & 10.19 & -1.51 \\
\hline Lee Shau Kee Bldg. & 319.74 & 275.89 & 275.36 & 8.81 & -0.24 \\
\hline Siu Loo & 122.42 & 287.23 & 275.79 & 7.93 & -0.23 \\
\hline Pi Ch'iu Bldg. & 23.54 & 337.80 & 278.88 & 28.34 & -2.9 \\
\hline
\end{tabular}

Table 2. Building Visibility Figures (Visual Distance and Angle) for the Experimental Site of Central Campus Square

The visual data computation result implies that the author cannot directly adopt others' hemisphere visual model into this research. As the result of the hilly topography feature of CUHK campus, from the result it is obviously noticed that some buildings are showing negative vertical visual angle in this site, such as Lady Shaw Bldg., Ho Sin-Hang Engineering Bldg. and William M W Mong Engineering Bldg. That is because these three buildings are below the horizon of central campus square. Furthermore, a few buildings are on the top of the hill, such as FYT Remote Sensing Science Building and water towers saw by the observer, also show a different situation from the case of plain cities, which are generating short visual distance and large visual angle. According to the result got for all visible buildings, a few efforts could be made such as the computation for the visual impacts for single buildings, which is usually applied in the figure of environmental pressures. Normally the pressure is thought to be proportional to the visual impacts of a single building, and also to the value of visual angle. The ranking of building pressure for the observer could be down by these sets of data source, from the value of visual angle in vertical and horizontal direction.

\subsection{Integration of Visual Data for Terrain and Buildings}

According to the data procession outputs for terrain and buildings, the integration work has been done between the two sources in visual distance and visual angle. The visual field for each experimental site is the main output of the integration, as it will be applied for the identification of space types and correlation study of human's feeling. As the definition of visual field is a distribution of visual distance and angle in 360 directions, the comparison work is necessary to integrate the data from terrain and building models. That is, for visual distance sources, only smaller one will be concerned in the integration, because it represent the visible border for the viewpoint; for visual angle sources, the larger figure of maxvertical one is thought to be the significant figure and used in visual field, since it represents the skyline of the visible area.

Currently, the author has applied the conversions in a few example sites and got some outputs. These representative sites are: Central Campus Square, Chung Chi Lotus Pond, United College Square and New Asia College Courtyard, which all locate inside CUHK campus. Similar with the method of DEM based 3D isovist (Morello and Ratti 2009), the figures of visual field can also be generated from the VGE platform, which is using a comparison method of visual angle in different directions. In this preliminary study, the visual fields for these 4 points mentioned above have been computed and the output could be referred from Figure 8 and Figure 9. Besides, a statistics work has been done for each experimental site to see if there are any features for the identification of its spatial type.
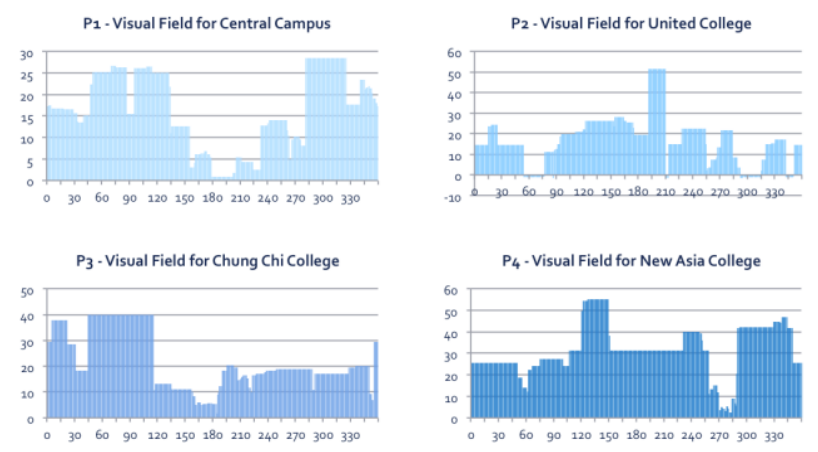

Figure 8. Visual Field for 4 Experimental Sites in the Preliminary Research

The graphs of visual field represents the distribution of visible visual angle from the observer's location, which tents to be similar with the skyline, although meets the rule of bigger when closer and smaller when farther. From the visual field graphs, a few environmental distinct features could be noticed, such as the water towers in both United College and New Asia College, which are showing distinct peaks and could be noticed from the graphs. But currently, only maximum of the vertical visual angle is used, because it is significant and marks the border between buildings and sky. The visual fields are more or less continuous with the value distributed in a few ranges. In this situation, if the statistics work is done for every 15-degree or 5degree for the processed result, a percentage distribution of vertical visual angle could be seen as following Figure 9.

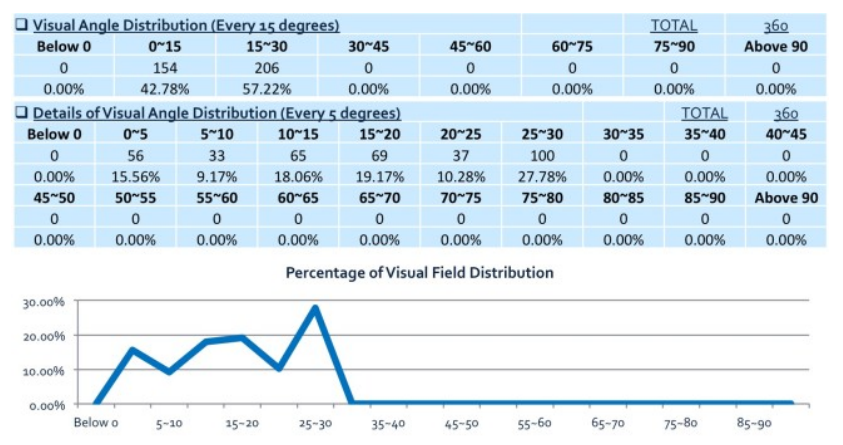

Figure 9. Visual Field Statistics for Central Campus Square, and Distribution of Visual Angles in 5-degree Ranges 
For similar space types, the distribution patterns should also be similar with each other. From this, the space pattern could be identified and applied for further analysis, such as the correlation between these figures and human's feeling. Take squares for instance, the distribution of the visual angle is strongly restricted in the range between $5^{\circ}$ and $30^{\circ}$ and mostly continuous and without any gaps; but for courtyards, the visual angle border has relatively higher values and ranges from $25^{\circ}$ to $50^{\circ}$. Although the locations are different, similar space types will have similar visual field distribution figures. As this is a preliminary study, more progresses are going to be found in future, and experiments will be taken in more locations to validate this assumption.

\section{REMARKS, CONCLUSIONS AND FUTURE PLAN}

This article is aiming at providing a new solution for the relationship study between the spaces and human's perception, and doing the 3D visibility analysis by using the VGE platform. According to the experiment in this article, a few conclusions have been drawn from the author's current research progresses. Firstly, this article introduced the method of 3D visibility analysis applying in the experimental sites with hilly topography, and tried to discover a few conditions not noticed before, such as the new space type - terraces. Then, to quantify the spatial information in the environment and make it measurable, the author used 3 features here as visual distance, visual angle and visual field, which could also be applied for the computation of openness, permeability, environmental pressure and visibility. To avoid the inaccuracy generated by the procession of DEM or TIN model only, in this experiment the author developed a method of terrain and structures integration, by using the data source from both DEM and building models. Furthermore, after getting the results of visual data for each single building, a ranking of building importance, pressure and impact could be done according to the possession of the visual angle size and distance, which is useful for us to understanding the cognition processes of the environment. At the end, the idea of visual field has been adopted in the identification of 4 space types - squares, courtyards, terraces and streets, depending on its enclosure level and continuity of visual field. These 4 types of spaces are showing different enclosure, permeability, environmental pressure and visibility from each other, and this point may be focused in the further research to find out more details about the relationship with human's perception.

At present this research approach still has several things to do. Vegetation is going to be led into as a factor and focused on its effect towards human's feeling in the certain environment. Moreover, due to the inexperience for building models applied in visibility analysis, the extraction method of visual angle for buildings is necessary to be improved to achieve better accuracy, as currently it only considered about the value in each vertex of the bounding box in this research. The correlation study between the spatial features and human's feeling are going to be carried on, and the transferring method from visual distance, visual angle and visual field to the human's perception figure as enclosure or permeability will be concerned in the future progress. Besides, proper validation work will take in the further research to ensure the practicality of this methodology. These goals are going to be achieved next to enrich the current research work of the author.

\section{ACKNOWLEDGEMENTS}

The work described in this paper is supported by National Natural Science Foundation of China (grant no. 41171146 and 41101370) and the Open Research Fund of Key Laboratory of Disaster Reduction and Emergency Response Engineering of the Ministry of Civil Affairs (grant no. LDRERE20120302). We also appreciate the detailed suggestions and comments from the editor and the anonymous reviewers.

\section{REFERENCE:}

Ashihara Y.. Design of Exterior Spaces. Shokokusha, Tokyo, Japan, 1975. (芦原義信、外部空間の設計、彰国社、1975 年 in Japanese)

Benedikt M. L. To take hold of space: isovists and isovist fields. Environment and Planning B, 1979, volume 6, pp. 47-65.

Bittermann M. Intelligent Design Objects (IDO): a cognitive approach for performance-based design. PhD Research in Delft University of Technology, 2009.

Fisher-Gewirtzman D., Wagner I. Spatial Openness as a Practical Metric for Evaluating Built-Up Environments. Environment and Planning B: Planning and Design, 2003, Vol. 30 issue 1 pp. 37-49.

Fisher-Gewirtzman D., Pinsly D. S., Wagner I. A., Burt M. View-oriented three-dimensional visibility analysis models for the urban environment. Urban Design International, 2005, volume 10, pp. 23-37.

Friedman D., Steed A., Slater M. Spatial Social Behavior in Second Life. IVA 2007, LNAI 4722, 2007, pp. 252-263.

Gould, P. On mental maps. In R. M. Downs and D. Stea (eds.) Image and Environment: Cognitive Mapping and Spatial Behaviour. London: Edward Arnold, 1973, pp. 182 - 220.

Kinicki J., Claypool M. Traffic Analysis of Avatars in Second Life, NOSSDAV'08 Braunschweig, Germany, 2008.

Leduc T., Tourre V., Woloszyn P., Miguet F. Measuring Surrounding Space to Assess the Pedestrian Visual Aperture Angle in the Urban Fabric: Toward a Kurtosis-Based Isovist Indicator. Information Fusion and Geographic Information Systems, 2011, Chapter 11, pp. 129-142.

Llobera M. Extending GIS-based visibility analysis: the concept of visualscapes. International Journal of Geographic Information Science, 2003, Volume 17, pp. 25-48.

Lynch K. Managing the Sense of Region. MIT Press, Cambridge, MA, USA, 1976.

Lynch K. A Theory of Good City Form. MIT Press, Cambridge, MA, USA, 1981.

Montgomery J. Making a City: Urbanity, Vitality and Urban Design. Journal of Urban Design Quarterly, 1998, No 53 (January) pp. 15-17. 
ISPRS Annals of the Photogrammetry, Remote Sensing and Spatial Information Sciences, Volume II-2/W1, ISPRS 8th 3DGeolnfo Conference \& WG II/2 Workshop, 27 - 29 November 2013, Istanbul, Turkey

Morello E., Ratti C. A digital image of the city: $3 D$ isovists in Lynch's urban analysis. Environment and Planning B: Planning and Design, 2009, volume 36(5), pp. 837-853.

Stamps III A. E. Isovists, enclosure, and permeability theory. Environment and Planning B: Planning and Design 2005, volume 32, pp. 735-762.

Stamps III A. E. Visual Permeability, Locomotive Permeability, Safety and Enclosure. Environment and Behavior, 2005, volume 37(5), pp. 587-619.

Suleiman W., Joliveau T., Favier E. 3D Urban Visibility Analysis with Vector GIS Data. Presented at the GISRUK, 2011, University of Portsmouth, UK, 27-29 avril 2011 April 26 (2011).

Tandy C. R. V. The isovist method of landscape survey. Methods of Landscape Analysis, Landscape Research Group, London, 1967, pp. 9-10

Turner A., Doxa M., O'Sullivan D., Penn A. From isovists to visibility graphs: a methodology for the analysis of architectural space. Environment and Planning B: Planning and Design, 2001, volume 28, pp. 103-121

Turner A., Penn A. Making isovists syntactic: isovist integration analysis. 2nd International Symposium on Space Syntax, Universidad de Brasilia, Brazil, April 1999.

Yang P. P., Putra S. Y., Li W. J. Viewsphere: a GIS-based 3D visibility analysis for urban design evaluation. Environment and Planning B: Planning and Design, 2007, volume 34, pp. 971-992. 